\title{
Traveling waves in a finite condensation rate model for steam injection
}

\author{
J. Bruining • C.J. van Duijn
}

Received: 24 May 2005 / Accepted: 25 August 2006 / Published online: 7 November 2006

(C) Springer Science + Business Media B.V. 2006

\begin{abstract}
Steam drive recovery of oil is an economical way of producing oil even in times of low oil prices and is used worldwide. This paper focuses on the onedimensional setting, where steam is injected into a core initially containing oil and connate water while oil and water are produced at the other end. A three-phase (oil, water, steam) hot zone develops, which is abruptly separated from the two-phase (oil + water) cold zone by the steam condensation front. The oil, water and energy balance equations (Rankine-Hugoniot conditions) cannot uniquely solve the system of equations at the steam condensation front. In a previous study, we showed that two additional constraints follow from an analysis of the traveling wave equation representing the shock; however, within the shock, we assumed local thermodynamic equilibrium. Here we extend the previous study and include finite condensation rates; using that appropriate scaling requires that the Peclet number and the Damkohler number are of the same order of magnitude. We give a numerical proof, using a colorcoding technique, that, given the capillary diff usion behavior and the rate equation, a unique solution can be obtained. It is proven analytically that the solution for large condensation rates tends to the solution obtained assuming local thermodynamic equilibrium. Computa-
\end{abstract}

\section{J. Bruining $(\bowtie)$}

Dietz Laboratory, Centre for Technical Geoscience, Delft University of Technology, Delft, The Netherlands e-mail: j.bruining@ta.tudelft.nl

C.J. van Duijn

Department of Mathematics and Computer Science,

Eindhoven University of Technology,

Eindhoven, The Netherlands

e-mail: c.j.v.Duijn@tue.nl tions with realistic values to describe the viscous and capillary effects show that the condensation rate can have a significant effect on the global saturation profile, e.g. the oil saturation just upstream of the steam condensation front.

Keywords finite condensation • steam injection • traveling wave equation

\section{Introduction}

Steam injection, as a method for enhanced oil recovery, received considerable attention in the petroleum engineering literature during the past decades $[11,16,32]$. Recently, there is renewed interest for the purpose of removing oil spills from the subsurface $[3,19,20,23$, $37,38]$. The processes involved are extremely complex and pose challenging questions concerning theory $[12,26,45,46]$, experiment $[13,24,43]$ and numerical modeling [1, 5, 8, 11, 29-31].

The theoretical study described in this paper builds on previous work [7, 39]. In [7], we considered a simplified model describing oil recovery by steam drive. The proposed model assumes small capillary forces and instantaneous condensation as a result of thermodynamic equilibrium. It has an upstream hot threephase (oil, water, steam) flow zone and a downstream cold two-phase (oil, water) flow zone. The upstream and downstream zones are separated by a relatively thin transition region, which is described by a (local) steam condensation/capillary diffusion model based on the ideas of Udell et al. [28, 40, 42]. In the limit of zero capillary forces, the transition region collapses to form a steam condensation front (SCF). Disregarding 
capillary pressure away from the condensation front, a $2 \times 2$ hyperbolic system [14, 15] arises for water and steam. This system cannot be solved uniquely without additional conditions at the SCF. To find these conditions, we studied traveling waves of the capillary model in the transition region [21, 22, 25, 27]. In [7], we investigated the effect of different capillary pressure behavior, the effect of gradual-versus-abrupt temperature decline from the steam temperature to ambient temperature and the effect of non-zero gas saturation at the SCF. In all these cases, we assumed local thermodynamic equilibrium. We found and made explicit that details of the transition model affect the global behavior of the steam displacement process. It is therefore of interest to investigate whether the global behavior also depends on the rate constant, i.e. if we drop the assumption of thermodynamic equilibrium and implement a condensation rate model. A finite reaction rate model is also preferred in numerical simulations. We expect that in the limit of large rate constants, the results for local equilibrium are retrieved. The aim of this paper is to investigate those two aspects.

In Section 2, we briefly describe the model and recall the model equations. The hyperbolic setting and a summary of previous results are given in Section 3 . In Section 4, we define the traveling wave problem and the method to obtain the unique solution for a given condensation model. The route to thermodynamic equilibrium is explained in Section 5 by sending the rate constant to infinity. We end in Section 6 with computations for some realistic cases and comparison to previous results.

\section{Finite rate condensation model}

\subsection{Physical considerations}

Oil displacement by steam drive through a porous medium is a complex physical process that is controlled by the steam condensation process and by viscous and capillary forces (see for instance Stewart \& Udell [40] and Wingard \& Orr [44]. Following ideas of Shutler [39], we proposed in [7] a one-dimensional model where all complexity is confined to a small transition region in which the condensation occurs and capillary forces act. The model describes the case of injecting steam in a linear core originally filled with oil and connate water. The porosity $\varphi$ and permeability $k$ are constant. We allow for temperature-dependent liquid viscosities except that we assume the steam viscosity to be independent of temperature because these viscosities are small anyway and the temperature depen- dence of $\mu_{g} \sim T^{0.6}$ is much smaller than for the liquid viscosities.

The core is horizontal and we disregard the effects of gravity. Transverse capillary pressure diffusion is sufficiently large to guarantee a uniform saturation over the cross-section. The core is positioned along the positive $x$-axis with flow from left to right, implying that all variables are functions of position $x$ and time $t$. The displacement is considered to occur at constant pressure, in the sense that we disregard flow-induced pressure gradient effects on the thermodynamic properties, reaction rates, fluid densities and viscosities. Therefore, the pressure does not explicitly enter in the model equations, but it determines the value of some parameters. The temperature dependence of the parameters is summarized in table 1 . The oil considered is dead oil; that is, it does not occur in the gas phase. Dissolution of liquid oil in water and vice versa is disregarded. The condensation occurs between an upstream threephase flow zone at steam temperature $T_{\mathrm{b}}$, where oil, water and steam are present, and a downstream twophase flow zone at the initial reservoir temperature $T_{\mathrm{o}}$, where water and oil are present. In the upstream and downstream regions, capillary forces are disregarded. Consequently, these regions are adequately described by an (extended) Buckley-Leverett approach. We use power-law relative permeabilities (both quadratic and fourth powers), as well as Stone I expressions [9].

All condensation occurs in a thin region called the steam condensation front (SCF). The constant travel speed of the SCF is determined from an energy balance that is decoupled from the mass balance equations. The decoupling is achieved by disregarding the effect of fluid content on the heat capacity $(\rho c)_{\mathrm{r}}$ of the porous medium. The velocity $v_{\mathrm{st}}$ is determined from a local heat balance, in which the heat released by the condensing steam impinging on the SCF is equal to the amount of heat necessary to warm up the reservoir (see Mandl and Volek [26]). The result is

$$
v_{s t}=\frac{\rho_{\mathrm{g}} \Delta H u_{\mathrm{inj}}}{(\rho c)_{\mathrm{r}}\left(T_{\mathrm{b}}-T_{\mathrm{o}}\right)} .
$$

The symbols appearing in this expression are explained in table 1.

Within the transition region, there is an interplay between viscous forces, capillary forces and the condensation process. In this paper, we use a finite rate condensation model. There are three dimensionless numbers involved in the processes that occur in the transition zone, i.e. the Peclet number for mass transport $(P e)$, the Peclet number for heat transport $\left(P e_{\mathrm{T}}\right)$ and the Damkohler number $(D a)$. These numbers indicate the ratio of phase transport by convection and 
Table 1 Summary of physical input parameters ${ }^{a}$.

\begin{tabular}{|c|c|c|c|}
\hline Physical quantity & Symbol & Value & Unit \\
\hline Characteristic length & $\mathrm{L}$ & 100 & $\mathrm{~m}$ \\
\hline Steam temperature & $T_{\mathrm{b}}$ & 486 & $\mathrm{~K}$ \\
\hline Reservoir temperature & $T_{\mathrm{o}}$ & 313 & $\mathrm{~K}$ \\
\hline Injection rate steam & $u_{\text {inj }}$ & $9.52 \times 10^{-4}$ & $\mathrm{~m}^{3} / \mathrm{m}^{2} / \mathrm{s}$ \\
\hline Steam viscosity & $\mu_{\mathrm{g}}$ & $1.63 \times 10^{-5}$ & $\mathrm{~Pa} \mathrm{~s}$ \\
\hline Oil viscosity at $T_{\mathrm{b}}$ & $\mu_{\mathrm{o}}\left(T_{\mathrm{b}}\right)$ & $2.45 \times 10^{-3}$ & Pa s \\
\hline Oil viscosity at $T_{\mathrm{o}}$ & $\mu_{\mathrm{o}}\left(T_{\mathrm{o}}\right)$ & 0.180 & Pa s \\
\hline Water viscosity at $T_{\mathrm{b}}$ & $\mu_{\mathrm{w}}\left(T_{\mathrm{b}}\right)$ & $1.30 \times 10^{-4}$ & $\mathrm{~Pa} \mathrm{~s}$ \\
\hline Water viscosity at $T_{\mathrm{o}}$ & $\mu_{\mathrm{W}}\left(T_{\mathrm{o}}\right)$ & $7.21 \times 10^{-4}$ & Pa s \\
\hline Reference viscosity & $\mu_{\mathrm{w}}^{*}$ & $5.0 \times 10^{-4}$ & $\mathrm{~Pa} \mathrm{~s}$ \\
\hline Brooks-Corey sorting factor & $\lambda_{\mathrm{s}}$ & 2 & - \\
\hline Rate constant & $q_{\mathrm{b}}$ & $10^{3}$ & $\mathrm{~s}^{-1}$ \\
\hline Enthalpy $\mathrm{H}_{2} \mathrm{O}(l)\left(T_{\mathrm{o}}\right) \rightarrow \mathrm{H}_{2} \mathrm{O}(g)\left(T_{1}\right)$ & $\Delta H$ & 2,636 & $\mathrm{~kJ} / \mathrm{kg}$ \\
\hline Effective heat capacity of rock & $(\rho c)_{\mathrm{r}}$ & 2,029 & $\mathrm{~kJ} / \mathrm{m}^{3} / \mathrm{K}$ \\
\hline Thermal coefficient & $\alpha$ & 0.017 & - \\
\hline Capillary diffusion constant & $D$ & $1.85 \times 10^{-7}$ & $\mathrm{~m}^{2} / \mathrm{s}$ \\
\hline Diffusion correction factor & $d$ & $10^{-2}$ & - \\
\hline Velocity SCF & $v_{\mathrm{st}}$ & $7.12 \times 10^{-5}$ & $\mathrm{~m} / \mathrm{s}$ \\
\hline Porosity & $\varphi$ & 0.38 & $\mathrm{~m}^{3} / \mathrm{m}^{3}$ \\
\hline Permeability & $k$ & $1.0 \times 10^{-12}$ & $\mathrm{~m}^{2}$ \\
\hline Interfacial tension & $\sigma$ & 0.03 & $\mathrm{~N} / \mathrm{m}$ \\
\hline Water density & $\rho_{\mathrm{W}}$ & 1,000 & $\mathrm{~kg} / \mathrm{m}^{3}$ \\
\hline Steam density & $\rho_{\mathrm{g}}$ & 10.2 & $\mathrm{~kg} / \mathrm{m}^{3}$ \\
\hline Connate water saturation & $S_{\mathrm{wc}}$ & 0.15 & $\mathrm{~m}^{3} / \mathrm{m}^{3}$ \\
\hline Residual gas saturation & $S_{\mathrm{gr}}$ & 0.0 & $\mathrm{~m}^{3} / \mathrm{m}^{3}$ \\
\hline Residual oil saturation & $S_{\text {or }}$ & 0.0 & $\mathrm{~m}^{3} / \mathrm{m}^{3}$ \\
\hline
\end{tabular}

${ }^{a}$ The values of the steam parameters in the table assume a steam pressure of 20 bars. Furthermore, the value of the thermal coefficient $\alpha$ is based on a thermal diffusivity of $9.85 \times 10^{-7}\left(\mathrm{~m}^{2} / \mathrm{s}\right)$. Note that this coefficient is proportional to the ratio of the capillary and thermal diffusivity.

diff usion, the ratio of heat convection and thermal conductivity and the ratio of the rate of convected phase transport and the condensation rate, respectively. In the model, we assume an instantaneous temperature drop from steam temperature to reservoir temperature as the steam saturation becomes zero. Furthermore, we assume that the Damkohler number $D a$ and the Peclet number $P e$ are of the same order of magnitude. We compare our results to those obtained in [7], where it was assumed that all steam condenses at a single point in the transition region, the actual (SCF). The rate of condensation is sufficiently fast so that, indeed, all condensation occurs in a small neighborhood of the SCF. Here "small" must be understood in a suitable dimensionless context. In the condensation zone, the temperature drops from steam temperature $T_{\mathrm{b}}$ to the original reservoir temperature $T_{\mathrm{o}}$, and steam condenses at a rate proportional to $\left(T_{\mathrm{b}}-T\right)$, where $T$ is the prevailing temperature. As long as there is steam, the condensation rate is proportional to the saturation $S_{\mathrm{g}}$. When the steam saturation is zero, the pores are fully saturated with water and oil, and the condensation rate becomes zero. This leads to the following expression for the mass condensation rate $q$

$$
q=\left\{\begin{array}{lc}
\rho_{\mathrm{g}} q_{\mathrm{b}} \frac{T_{\mathrm{b}}-T}{T_{\mathrm{b}}-T_{\mathrm{o}}} S_{\mathrm{g}} & \text { for } \quad T \leq T_{\mathrm{b}}, \quad 0<S_{\mathrm{g}} \leq 1-S_{\mathrm{wc}}, \\
0 & \text { otherwise, }
\end{array}\right.
$$

where $q_{\mathrm{b}}$ is the condensation rate parameter. As in [7], we assume that the temperature distribution can be determined independently from the condensation process. In fact, in [7], we distinguished between an exponential decline and a stepwise decline. In this paper, we confine ourselves to the stepwise decline. Hence, the temperature is discontinuous and is given by

$T= \begin{cases}T_{\mathrm{b}} & x<v_{\mathrm{st}} t \\ T_{\mathrm{o}} & x \geq v_{\mathrm{st}} t .\end{cases}$

The phase densities $\rho_{\alpha}(\alpha=\mathrm{w}, \mathrm{o}, \mathrm{g})$ are assumed to be constant throughout this paper. 


\subsection{Model equations}

The mass balance equations for water, steam and oil read

$\left\{\varphi \frac{\partial\left(\rho_{\mathrm{w}} S_{\mathrm{w}}\right)}{\partial t}+\frac{\partial\left(\rho_{\mathrm{w}} u_{\mathrm{w}}\right)}{\partial x}=q\right.$,

$\left\{\varphi \frac{\partial\left(\rho_{\mathrm{g}} S_{\mathrm{g}}\right)}{\partial t}+\frac{\partial\left(\rho_{\mathrm{g}} u_{\mathrm{g}}\right)}{\partial x}=-q\right.$,

$\left\{\varphi \frac{\partial\left(\rho_{\mathrm{o}} S_{\mathrm{o}}\right)}{\partial t}+\frac{\partial\left(\rho_{\mathrm{o}} u_{\mathrm{o}}\right)}{\partial x}=0\right.$.

where $q$ is given by expression (1). The phase saturations satisfy

$0 \leq S_{\mathrm{wc}} \leq S_{\mathrm{w}} \leq 1,0 \leq S_{\mathrm{g}}, S_{\mathrm{o}} \leq 1-S_{\mathrm{wc}}$.

In other words, we assume that there is no residual oil and gas in the system. We use Darcy's law for multiphase flow to express all phase velocities $u_{\alpha}$ in terms of the total velocity $u$ and the capillary pressures (see [1] and also [10, 18, 33, 34]. This gives

$\left\{u_{\mathrm{w}}=u f_{\mathrm{w}}+\lambda_{\mathrm{o}} f_{\mathrm{w}} \frac{\partial p_{\mathrm{c}, \mathrm{ow}}}{\partial x}+\lambda_{\mathrm{g}} f_{\mathrm{w}} \frac{\partial p_{\mathrm{c}, \mathrm{gw}}}{\partial x}\right.$,

$\left\{u_{\mathrm{g}}=u f_{\mathrm{g}}-\lambda_{\mathrm{o}} f_{\mathrm{g}} \frac{\partial p_{\mathrm{c}, \mathrm{go}}}{\partial x}-\lambda_{\mathrm{w}} f_{\mathrm{g}} \frac{\partial p_{\mathrm{c}, \mathrm{gw}}}{\partial x}\right.$,

$\left\{u_{\mathrm{o}}=u f_{\mathrm{o}}-\lambda_{\mathrm{w}} f_{\mathrm{o}} \frac{\partial p_{\mathrm{c}, \mathrm{ow}}}{\partial x}+\lambda_{\mathrm{g}} f_{\mathrm{o}} \frac{\partial p_{\mathrm{c}, \mathrm{go}}}{\partial x}\right.$.

Here

$u=u_{\mathrm{w}}+u_{\mathrm{o}}+u_{\mathrm{g}}$,

$p_{c, \alpha \beta}$ is the capillary pressure, being the pressure difference between phase $\alpha$ and phase $\beta$, and $f_{\alpha}$ is the fractional flow function

$f_{\alpha}=\frac{\lambda_{\alpha}}{\lambda_{\mathrm{o}}+\lambda_{\mathrm{w}}+\lambda_{\mathrm{g}}}$.

Further, $\lambda_{\alpha}$ denotes the mobility of phase $\alpha$, given by $\lambda_{\alpha}=\frac{k k_{r a}}{\mu_{\alpha}}$,

where $k_{\mathrm{r} \alpha}$ is the relative permeability (fourth power of the effective saturations) and $\mu_{\alpha}$ is the phase viscosity. Because water and oil experience different temperatures, their viscosities may vary significantly across the SCF. Realistic values $[4,35,41]$ are given in table 1 . In later sections, we use the notation $f_{\alpha}^{ \pm}$, where $f_{\alpha}^{-}$ denotes the fractional flow function in the hot steam zone and $f_{\alpha}^{+}$in the cold oil zone.

Because

$\sum_{\alpha} S_{\alpha}=\sum_{\alpha} f_{\alpha}=1$ and $u=\sum_{\alpha} u_{\alpha}$, we can eliminate, for instance, $S_{\mathrm{o}}$ from the equations. Further, summing Eqs. (6), (7) and (8) and using Eq. (9), we find

$\frac{\partial u}{\partial x}=-\frac{1}{\rho_{\mathrm{g}}}\left(1-\frac{\rho_{\mathrm{g}}}{\rho_{\mathrm{w}}}\right) q=-q_{\mathrm{b}}\left(1-\frac{\rho_{\mathrm{g}}}{\rho_{\mathrm{w}}}\right)\left(\frac{T_{\mathrm{b}}-T}{T_{\mathrm{b}}-T_{\mathrm{o}}}\right) S_{\mathrm{g}}$.

Thus, our primary variables are $u, S_{\mathrm{w}}$ and $S_{\mathrm{g}}$ for which we have Eqs. (11), (2+6) and (3+7). Injecting only steam from the left at $x=0$ and having only oil and connate water present at $t=0$ require the boundary/initial conditions:

$u(0, t)=u_{\mathrm{inj}}, S_{\mathrm{w}}(0, t)=S_{\mathrm{wc}}$, and $S_{\mathrm{g}}(0, t)=1-S_{\mathrm{wc}}$,

for all $t>0$ and

$S_{\mathrm{w}}(x, 0)=S_{\mathrm{wc}}$ and $S_{\mathrm{g}}(x, 0)=0$,

for all $x>0$. In (12), $u_{\text {inj }}$ denotes the injection velocity of the steam.

We want to write the Darcy velocities $u_{\mathrm{w}}$ and $u_{\mathrm{g}}$ in terms of capillary diffusion involving $S_{\mathrm{w}}$ and $S_{\mathrm{g}}$ only. For this purpose, we note that

$p_{\mathrm{c}, \mathrm{gw}}=p_{\mathrm{g}}-p_{\mathrm{w}}=p_{\mathrm{c}, \mathrm{go}}+p_{\mathrm{c}, \mathrm{ow}}$,

where $p_{\mathrm{c}, \text { ow }}=p_{\mathrm{c}, \text { ow }}\left(S_{\mathrm{w}}\right)$ is a strictly decreasing function of the water saturation and where $p_{\mathrm{c}, \mathrm{go}}=p_{\mathrm{c}, \mathrm{ow}}\left(1-S_{\mathrm{g}}\right)$ is a strictly increasing function of the gas saturation. For instance, in [7], we considered the Brooks-Corey expression (see also [2, 6, 36]),

$p_{\mathrm{c}, \text { ow }}=\frac{\sigma}{2} \sqrt{\frac{\varphi}{k}}\left(\frac{\frac{1}{2}-S_{\mathrm{wc}}}{1-S_{\mathrm{wc}}}\right)^{1 / \lambda_{\mathrm{s}}}\left(\frac{S_{\mathrm{w}}-S_{\mathrm{wc}}}{1-S_{\mathrm{wc}}}\right)^{-1 / \lambda_{\mathrm{s}}}$,

where $\lambda_{\mathrm{s}}>0$ is the sorting factor.

Using these observations, we obtain

$u_{\mathrm{w}}=u f_{\mathrm{w}}-\mathcal{D}_{\mathrm{ww}} \frac{\partial S_{\mathrm{w}}}{\partial x}-\mathcal{D}_{\mathrm{wg}} \frac{\partial S_{\mathrm{g}}}{\partial x}$,

$u_{\mathrm{g}}=u f_{\mathrm{g}}-\mathcal{D}_{\mathrm{gw}} \frac{\partial S_{\mathrm{w}}}{\partial x}-\mathcal{D}_{\mathrm{gg}} \frac{\partial S_{\mathrm{g}}}{\partial x}$,

where

$\mathcal{D}_{\mathrm{ww}}=-\left(\lambda_{\mathrm{o}}+\lambda_{\mathrm{g}}\right) f_{\mathrm{w}} \frac{\mathrm{d} p_{\mathrm{c}, \mathrm{ow}}}{\mathrm{d} S_{\mathrm{w}}}>0$,

$\mathcal{D}_{\mathrm{wg}}=-\lambda_{\mathrm{g}} f_{\mathrm{w}} \frac{\mathrm{d} p_{\mathrm{c}, \mathrm{go}}}{\mathrm{d} S_{\mathrm{g}}}<0$,

$\mathcal{D}_{\mathrm{gw}}=\lambda_{\mathrm{w}} f_{\mathrm{g}} \frac{\mathrm{d} p_{\mathrm{c}, \mathrm{ow}}}{\mathrm{d} S_{\mathrm{w}}}<0$,

$\mathcal{D}_{\mathrm{gg}}=\left(\lambda_{\mathrm{o}}+\lambda_{\mathrm{w}}\right) f_{\mathrm{g}} \frac{\mathrm{d} p_{\mathrm{c}, \mathrm{go}}}{\mathrm{d} S_{\mathrm{g}}}>0$. 
Except in Section 6, where we work out a realistic case based on expression (14), we consider throughout this paper

$\mathcal{D}_{\mathrm{ww}}=\mathcal{D}_{\mathrm{gg}}=\mathcal{D}=$ constant,

$\mathcal{D}_{\text {wg }}=\mathcal{D}_{\mathrm{gw}}=0$,

where the constant diffusivity is given by

$\mathcal{D}=\frac{\sigma \sqrt{\varphi k}}{\mu_{\mathrm{w}}^{*}} d$.

Here $\mu_{\mathrm{w}}^{*}$ denotes a characteristic water viscosity, e.g. $\mu_{\mathrm{w}}\left(T_{\mathrm{o}}\right)$ and $d$ accounts for the effect of the water relative permeability and the functional relation of the capillary pressure.

\subsection{Rescaled equations}

We rewrite the equations in dimensionless form by setting

$$
\begin{gathered}
S_{\mathrm{w}}:=\frac{S_{\mathrm{w}}-S_{\mathrm{wc}}}{1-S_{\mathrm{wc}}}, S_{\mathrm{o}}:=\frac{S_{\mathrm{o}}}{1-S_{\mathrm{wc}}}, S_{\mathrm{g}}:=\frac{S_{\mathrm{g}}}{1-S_{\mathrm{wc}}}, \\
T:=\frac{T-T_{\mathrm{o}}}{T_{\mathrm{b}}-T_{\mathrm{o}}}, u:=\frac{u}{u_{\mathrm{inj}}}, x:=\frac{x}{L}, t:=\frac{u_{\mathrm{inj}} t}{\varphi L},
\end{gathered}
$$

where $L$ represents a characteristic length of the problem, for instance, the distance between injection and production point. Introducing the reciprocal Peclet number $\varepsilon:=\mathcal{D} /\left(u_{\text {inj }} L\right)$ and the dimensionless rate constant $r:=q_{\mathrm{b}} \mathcal{D} / u_{\mathrm{inj}}^{2}$, we find

$\frac{\partial u}{\partial x}=-\frac{r}{\varepsilon}(1-\alpha)(1-T) S_{\mathrm{g}}$,

$\frac{\partial S_{\mathrm{w}}}{\partial t}+\frac{\partial u f_{\mathrm{w}}}{\partial x}=\alpha \frac{r}{\varepsilon}(1-T) S_{\mathrm{g}}+\varepsilon \frac{\partial^{2} S_{\mathrm{w}}}{\partial x^{2}}$,

$\frac{\partial S_{\mathrm{g}}}{\partial t}+\frac{\partial u f_{\mathrm{g}}}{\partial x}=-\frac{r}{\varepsilon}(1-T) S_{\mathrm{g}}+\varepsilon \frac{\partial^{2} S_{\mathrm{g}}}{\partial x^{2}}$.

Here $\alpha=\rho_{\mathrm{g}} / \rho_{\mathrm{w}}$. Generally, $\alpha<<1$. The boundary and initial conditions follow from (12) and (13). They read

$u(0, t)=1, S_{\mathrm{w}}(0, t)=0, S_{\mathrm{g}}(0, t)=1$ for all $t>0$

and

$S_{\mathrm{w}}(x, 0)=S_{\mathrm{g}}(x, 0)=0 \quad$ for all $x>0$.

The scaled temperature $T=T(x, t)$ is discontinuous at the SCF with

$T= \begin{cases}1 & x<v t, \\ 0 & x \geq v t,\end{cases}$

where $v=v_{\mathrm{st}} / u_{\mathrm{inj}}$.
Remark 2.1 Direct scaling of Eq. (11) gives

$\frac{\partial u}{\partial x}=-\frac{q_{\mathrm{b}} L}{u_{\mathrm{inj}}}(1-\alpha)(1-T) S_{\mathrm{g}}$

with $\frac{q_{\mathrm{b}} L}{u_{\text {inj }}}$ being the dimensionless rate parameter (Damkohler number). To balance terms in the equations, in particular after the additional blow-up $x:=$ $x / \varepsilon$, we need

$\frac{q_{\mathrm{b}} L}{u_{\mathrm{inj}}}=\frac{r}{\varepsilon}, \quad$ with $r=O(1)$ as $\varepsilon \downarrow 0$.

Realistic numbers from table 1 give:

$\frac{q_{\mathrm{b}} L}{u_{\mathrm{inj}}} \sim 10^{8}, r \sim 10^{2}$ and $\varepsilon \sim 10^{-6}$.

\section{Hyperbolic setting and previous results}

When the scale $L$ of the problem is such that $\mathcal{D}<<$ $u_{\text {inj }} L$, and thus $\varepsilon<<1$, one expects that Eqs. (20)(22) reduce to a hyperbolic system, similar to the one studied in [7], in which all steam condenses at the SCF, i.e.

$\frac{\partial u}{\partial x}=-(1-\alpha) \Lambda \delta(x-v t)$,

$$
\begin{aligned}
& \frac{\partial S_{\mathrm{w}}}{\partial t}+\frac{\partial u f_{\mathrm{w}}}{\partial x}=\alpha \Lambda \delta(x-v t), \\
& \frac{\partial S_{\mathrm{g}}}{\partial t}+\frac{\partial u f_{\mathrm{g}}}{\partial x}=-\Lambda \delta(x-v t)
\end{aligned}
$$

Here $\Lambda$ is the condensation rate that originates from the condensation terms in Eqs. (20)-(22) and $\delta$ the Dirac measure at $x=v t$. Below we make this precise for traveling wave solutions.

In [7], we showed that a solution of the system (26)-(28), satisfying (23) and (24) can only be a fast rarefaction with $u=1$ in the steam region $\{x<v t\}$, with a shock at the SCF $\{x=v t\}$, and with two-phase Buckley-Leverett behavior in the cold region $\{x>v t\}$ where $S_{\mathrm{g}}=0$ (see figure 1).

We also showed that to obtain a uniquely constructed solution, a local transition model at the SCF must be introduced. In the hyperbolic setting, there are four unknowns at the SCF: the water and gas saturation $\left(S_{\mathrm{w}}^{-}, S_{\mathrm{g}}^{-}\right)$on the left, and the water saturation $S_{\mathrm{w}}^{+}$and Darcy velocity $u^{+}$on the right. Counting the number of equations between them, we have from mass conservation two Rankine-Hugoniot conditions

(RH) $\left\{\begin{aligned} f_{\mathrm{w}}^{-}-v S_{\mathrm{w}}^{-}+\alpha \Lambda & =u^{+} f_{\mathrm{w}}^{+}-v S_{\mathrm{w}}^{+}, \\ f_{\mathrm{g}}^{-}-v S_{\mathrm{g}}^{-}-\Lambda & =0,\end{aligned}\right.$ 


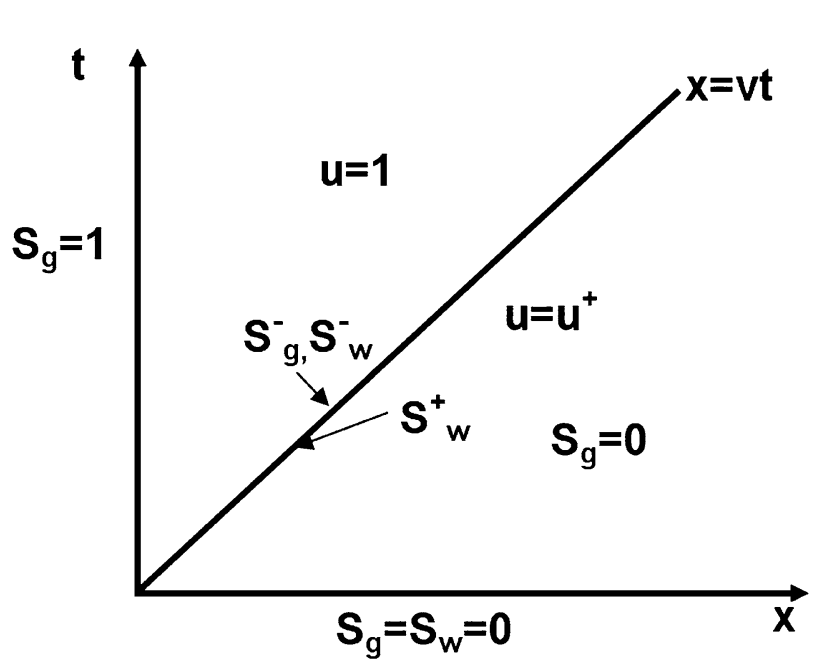

(a)

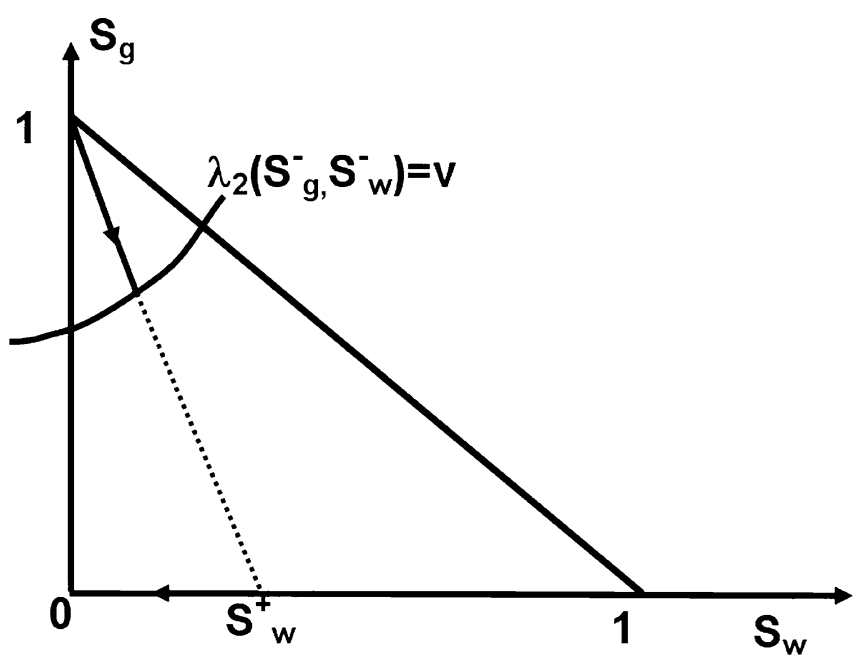

(b)

Figure 1 Water and gas saturations in hyperbolic model [(26)-(28)]. (a) Saturations in the (x,t) plane with unknowns $\left(S_{\mathrm{w}}^{-}, S_{\mathrm{g}}^{-}, S_{\mathrm{w}}^{+}, u^{+}\right)$ at the steam condensation front. (b) The saturation path in the $\left(S_{\mathrm{w}}, S_{\mathrm{g}}\right)$ plane.

with $\Lambda=\left(1-u^{+}\right) /(1-\alpha)$. A third equation follows from the condition that the fast rarefaction has to match up with the velocity of the SCF. This gives the entropy condition

$$
\text { (E) } \quad \lambda_{2}\left(S_{\mathrm{w}}^{-}, S_{\mathrm{g}}^{-}\right)=v \text {, }
$$

where $\lambda_{2}$ denotes the largest eigenvalue at $\left(S_{\mathrm{w}}^{-}, S_{\mathrm{g}}^{-}\right)$of the Jacobian matrix of the saturation flux $\left(f_{\mathrm{w}}, f_{\mathrm{g}}\right)$. The missing fourth relation follows from a traveling wave analysis of the transition model. In fact, the existence condition for a traveling wave, giving the shock its viscous profile, enabled us to construct a unique shock solution. We considered several local transition models, all having instantaneous condensation, and investigated their influence on the global solution through the shock condition at the SCF.

The main purpose of this paper is to understand the finite rate condensation model proposed in Section 2. We do this by analyzing traveling wave solutions of Eqs. (20)-(22). Such solutions allow us to quantify the role of the rate parameter $r$ and can be used as a building block in the construction of a unique shock solution.

In the finite rate condensation model, the rate constant is $r / \varepsilon$, where $r=O(1)$ and $\varepsilon$ is small. This is chosen to balance terms in the equations. To see this, we set

$\eta=\frac{x-v t}{\varepsilon}$

and consider the waves

$S_{i}=S_{i}(\eta) \quad(i=\mathrm{w}, \mathrm{g}) \quad$ and $\quad u=u(\eta)$.
Because of Eq. (25), we have $T=1-H(\eta)$, where $H$ denotes the Heaviside function

$$
H(\eta)= \begin{cases}0 & \eta<0 \\ 1 & \eta>0\end{cases}
$$

Substitution of Eqs. (31) and (32) into Eqs. (20)-(22) results in the system of ordinary differential equations

$\left.\begin{array}{l}u^{\prime}=-(1-\alpha) r(1-T) S_{\mathrm{g}}, \\ -v S_{\mathrm{w}}^{\prime}+\left(u f_{\mathrm{w}}\right)^{\prime}=\alpha r(1-T) S_{\mathrm{g}}+S_{\mathrm{w}}^{\prime \prime}, \\ -v S_{\mathrm{g}}^{\prime}+\left(u f_{\mathrm{g}}\right)^{\prime}=-r(1-T) S_{\mathrm{g}}+S^{\prime \prime}{ }_{\mathrm{g}},\end{array}\right\}$

where the primes denote differentiation with respect to $\eta$. Note that $\varepsilon$ has disappeared from the formulation and that the domain of the equations, for $\varepsilon \downarrow 0$, ranges from $\eta \rightarrow-\infty$ to $\eta \rightarrow+\infty$. As in [7] we impose the boundary conditions

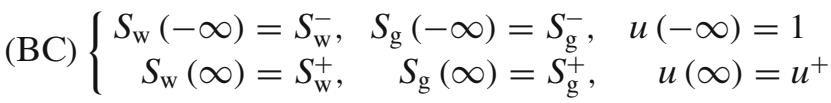

that satisfy $(\mathrm{RH})$, with $\Lambda$ appropriately chosen, and (E) .

Suppose, for the moment, that a traveling wave exists and that the decay of $S_{\mathrm{g}}(\eta) \rightarrow 0$ as $\eta \rightarrow \infty$ is such that

$$
\int_{0}^{\infty} S_{g}(\eta) \mathrm{d} \eta<\infty
$$

and

$\lim _{\varepsilon \downarrow 0} \frac{1}{\varepsilon} S_{\mathrm{g}}\left(\frac{\delta}{\varepsilon}\right)=0 \quad$ for every $\delta>0$. 
Clearly, for $i=\mathrm{w}, \mathrm{g}$,

$S_{i}^{\varepsilon}(x, t)=S_{i}\left(\frac{x-v t}{\varepsilon}\right) \quad$ and $\quad u^{\varepsilon}(x, t)=u\left(\frac{x-v t}{\varepsilon}\right)$

are solutions of Eqs. (20)-(22). In these equations, the condensation terms satisfy for any $t>0$

$$
\begin{aligned}
& \frac{r}{\varepsilon} \int_{\mathbb{R}}(1-T(x, t)) S_{\mathrm{g}}^{\varepsilon}(x, t) \mathrm{d} x= \\
& \frac{r}{\varepsilon} \int_{v t}^{\infty} S_{\mathrm{g}}^{\varepsilon}(x, t) \mathrm{d} x=r \int_{0}^{\infty} S_{\mathrm{g}}(\eta) \mathrm{d} \eta
\end{aligned}
$$

for all $\varepsilon>0$, and

$\lim _{\varepsilon \downarrow 0} \frac{r}{\varepsilon}(1-T(x, t)) S_{\mathrm{g}}^{\varepsilon}(x, t)=0 \quad$ for all $x \neq v t$.

Hence

$\lim _{\varepsilon \downarrow 0} \frac{r}{\varepsilon}(1-T(x, t)) S_{\mathrm{g}}^{\varepsilon}(x, t)=\Lambda \delta(x-v t)$,

where

$\Lambda=\Lambda(r):=r \int_{0}^{\infty} S_{g}(\eta) \mathrm{d} \eta$.

Remark 3.1 Conditions (34) and (35) follow directly from the construction of a solution. In Section 4, we show that in the $\left(u, S_{\mathrm{w}}, S_{\mathrm{g}}\right)$-space, the boundary point $\left(u^{+}, S_{\mathrm{w}}^{+}, 0\right)$ is a saddle with two positive and one negative eigenvalues. The latter provides exponential decay from which Eqs. (34) and (35) directly follow.

In the saturation equations from Eq. (33), we substitute

$r(1-T) S_{\mathrm{g}}=-\frac{1}{(1-\alpha)} u^{\prime}$.

Integrating the resulting expressions gives

$(\mathrm{TW})\left\{\begin{aligned} u^{\prime} & =-(1-\alpha) r(1-T) S_{\mathrm{g}}, \\ S_{\mathrm{w}}^{\prime} & =u f_{\mathrm{w}}-v S_{\mathrm{w}}+\frac{\alpha}{1-\alpha} u-\left(f_{\mathrm{w}}^{-}-v S_{\mathrm{w}}^{-}+\frac{\alpha}{1-\alpha}\right), \\ S_{\mathrm{g}}^{\prime} & =u f_{\mathrm{g}}-v S_{\mathrm{g}}-\frac{1}{1-\alpha} u-\left(f_{\mathrm{g}}^{-}-v S_{\mathrm{g}}^{-}-\frac{1}{1-\alpha}\right),\end{aligned}\right.$

with $-\infty<\eta<\infty$.

We analyze this dynamical system in the next section. To emphasize the construction and to avoid technical details, we consider the rather academic case in which the viscosities do not depend on temperature and in which the viscosity ratios are unity. Taking in addition quadratic relative permeabilities, the fractional flow functions simplify to

$f_{i}=f_{i}\left(S_{\mathrm{w}}, S_{\mathrm{g}}\right)=\frac{S_{i}^{2}}{S_{\mathrm{w}}^{2}+S_{\mathrm{g}}^{2}+\left(1-S_{\mathrm{w}}-S_{\mathrm{g}}\right)^{2}}$

and

$f_{i}^{ \pm}=f_{i}\left(S_{\mathrm{w}}^{ \pm}, S_{\mathrm{g}}^{ \pm}\right)$.

The results for a realistic case, with temperaturedependent viscosities, a large viscosity contrast and different relative permeabilities, will be presented and discussed in Section 6.

\section{Construction of shocks by means of traveling waves}

In this section, we explain the construction of a unique solution satisfying the simplified dynamic system (TW), with fractional flow functions given by Eqs. (37) and (38), subject to boundary conditions (BC) satisfying the constraints of Rankine-Hugoniot ( $\mathrm{RH})$ and entropy (E). The construction uses a shooting argument in the three-dimensional $\left(u, S_{\mathrm{w}}, S_{\mathrm{g}}\right)$-space.

Because

$S_{\mathrm{w}}+S_{\mathrm{g}}=1-S_{\mathrm{o}} \leq 1$ and $u^{\prime} \leq 0$,

we expect that a solution is confined to the domain

$$
\mathcal{R}=\left[u^{+}, 1\right] \times \mathbf{T}
$$

where $\mathbf{T}$ denotes the saturation triangle

$$
\mathbf{T}=\left\{\left(S_{\mathrm{w}}, S_{\mathrm{g}}\right): S_{\mathrm{w}}, S_{\mathrm{g}} \geq 0 \text { and } S_{\mathrm{w}}+S_{\mathrm{g}} \leq 1\right\}
$$

As in [7], the solution of the hyperbolic system (26)(28), satisfying boundary and initial conditions (23 and $24)$, respectively, follows a path as $A E$ in $\mathcal{R}$. This is

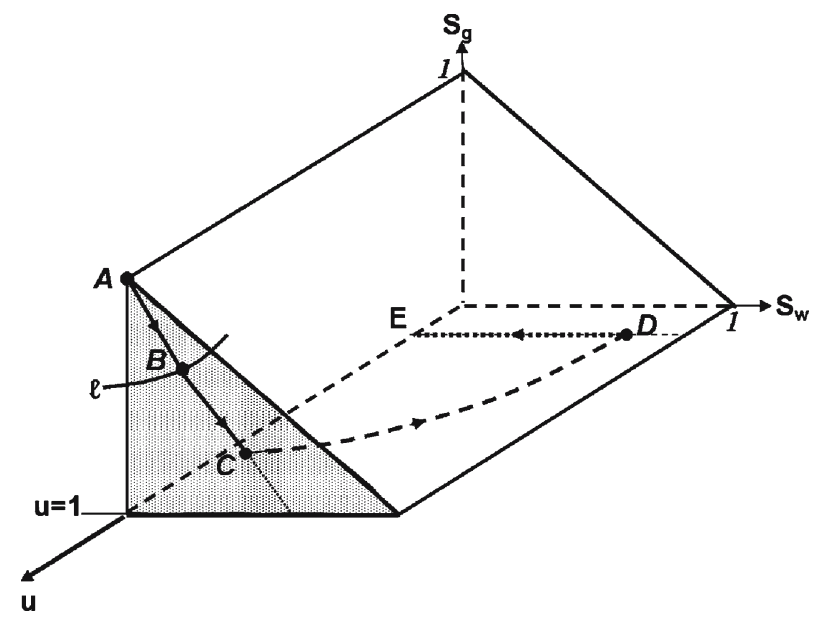

Figure 2 Orbit in $\left(u, S_{\mathrm{w}}, S_{\mathrm{g}}\right)$ space. 
sketched in figure 2. Here $A$ denotes the boundary condition $(1,0,1)$. The part $A B$ reflects the fast rarefaction in the steam region where $T=1$ and thus $u=1$. The point $B=\left(1, S_{\mathrm{w}}^{-}, S_{\mathrm{g}}^{-}\right) \in l$, where

$l=\left\{\left(u, S_{\mathrm{w}}, S_{\mathrm{g}}\right): u=1\right.$ and $\left.\lambda_{2}\left(S_{\mathrm{w}}, S_{\mathrm{g}}\right)=v\right\}$

Thus, for points in $l$, the speed of the fast rarefaction and the SCF coincide. With respect to the twodimensional triangle $\{u=1\} \times \mathbf{T}$, the point $B$ is a nonhyperbolic saddle with eigenvalues $\left(\right.$ at $\left(S_{\mathrm{w}}^{-}, S_{\mathrm{g}}^{-}\right)$of the Jacobian matrix of the vector $\left.\left(f_{\mathrm{w}}-v S_{\mathrm{w}}, f_{\mathrm{g}}-v S_{\mathrm{g}}\right)\right)$

$e_{1}<e_{2}=\lambda_{2}-v=0$

The part $B C D$ reflects the traveling wave as the viscous profile of the shock from $B$ to $D=\left(u^{+}, S_{\mathrm{w}}^{+}, 0\right)$. Because $T(\eta)=1$ for $\eta<0$, the part of the traveling wave with $-\infty<\eta<0$ has $u=1$ and is therefore confined to the face triangle $\{u=1\} \times \mathbf{T}$. At $C$, the temperature drops from boiling point to reservoir temperature implying that $T(\eta)=0$ for $\eta>0$. The path or orbit representing the solution now moves into the domain $\mathcal{R}$ with strictly decreasing $u$. At $D$, all steam has condensed. A two-phase Buckley-Leverett finally connects $D$ to the initial condition $E=\left(u^{+}, 0,0\right)$, with only movable oil being present.

The aim is now to show that for given $\alpha \in(0,1)$ and $v, r>0$, being the only parameters in the simplified problem, there exists a unique solution of (TW) which flows from $B \in l$ as $\eta \rightarrow-\infty$, through $C$ at $\eta=0$, to $D$ as $\eta \rightarrow \infty$. In this solution, $B$ and $D$ are related by conditions (RH) .

We first consider the construction for $\eta<0$. Because $u(\eta)=1$ for all $\eta<0$, we drop $u$ from the notation. With reference to figure 3 , let $S_{\mathrm{w}}^{\max }$ denote the maximum water saturation for which $\left(S_{\mathrm{w}}, S_{\mathrm{g}}\right) \in l$ in the saturation triangle $\mathbf{T}$. For $N \in \mathbb{N}$ sufficiently large, let

$S_{\mathrm{w}}^{-}(n)=\frac{n}{N} S_{\mathrm{w}}^{\max } \quad n=0,1,2, \ldots N$,

denote a uniform partition of the interval $\left[0, S_{\mathrm{w}}^{\max }\right]$ and let $B(n):=\left(S_{\mathrm{w}}^{-}(n), S_{\mathrm{g}}^{-}(n)\right)$ denote the corresponding partition of $l$. For each $n \in\{0,1, \ldots N\}$, we determine at $B(n)$ the eigenvector $\vec{e}_{2}$ corresponding to the eigenvalue $e_{2}=0$. This vector is indicated in figure 3 . In its direction, we solve the two saturation equations from (TW) with $u=1 \mathrm{kept}$ fixed. The corresponding solution is represented by the orbit $\Gamma(n)$ in figure 3 . It reaches $\left(S_{\mathrm{w}}=S_{\mathrm{w}}^{0}(n), S_{\mathrm{g}}=0\right)$ at finite $\eta$. Later on, we shall redefine $\eta$ such that $\eta=0$ corresponds to point $C$ in figure 2 .

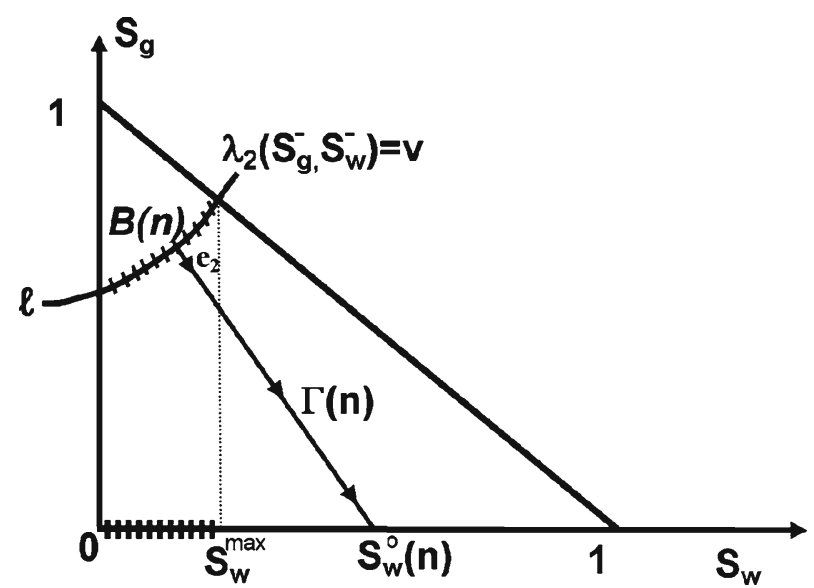

Figure 3 Construction of solution for $\eta<0$, with $u=1$. Here $B(n)$ denotes the point $\left(S_{\mathrm{w}}^{-}(n), S_{\mathrm{g}}^{-}(n)\right)$.

Next we construct the solution for $\eta>0$. For given $n \in\{0, \ldots, N\}$, we first determine from $(\mathrm{RH})$ the corresponding water saturation $S_{\mathrm{w}}^{+}(n)$ and the downstream fluid discharge $u^{+}(n)$. This yields the point $D(n)=$ $\left\{u^{+}(n), S_{\mathrm{w}}^{+}(n), 0\right\}$ as indicated in figure 4 .

Again we use (RH), now to put equations (TW), for $\eta>0$, in the form

$\left(\mathrm{TW}_{+}\right)\left\{\begin{aligned} u^{\prime} & =-(1-\alpha) r S_{\mathrm{g}}, \\ S_{\mathrm{w}}^{\prime} & =u f_{\mathrm{w}}-v S_{\mathrm{w}}+\frac{\alpha}{1-\alpha} u-F_{\mathrm{w}}^{+}(n), \\ S_{\mathrm{g}}^{\prime} & =u f_{\mathrm{g}}-v S_{\mathrm{g}}-\frac{1}{1-\alpha} u-F_{\mathrm{g}}^{+}(n),\end{aligned}\right.$

where

$F_{\mathrm{w}}^{+}(n)=u^{+}(n) f_{\mathrm{w}}\left(S_{\mathrm{w}}^{+}(n), 0\right)-v S_{\mathrm{w}}^{+}(n)+\frac{\alpha}{1-\alpha} u^{+}(n)$,

$F_{\mathrm{g}}^{+}(n)=-\frac{1}{1-\alpha} u^{+}(n)$.

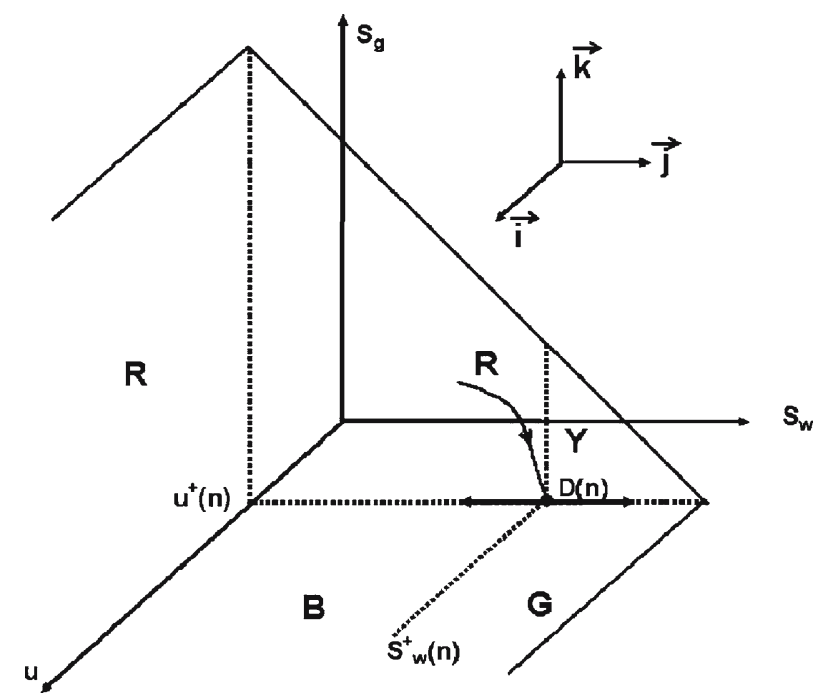

Figure 4 Sketch of exit sets near $D(n)=\left(u^{+}(n), S_{\mathrm{w}}^{+}(n), 0\right)$. 
We first determine the nature of the stationary point $D(n)$. From the Jacobian matrix at $D(n)$, we find one negative and two positive eigenvalues:

$$
\begin{aligned}
& \lambda_{1}=-\frac{v}{2}-\frac{1}{2} \sqrt{v^{2}+4 r}<0 \\
& \lambda_{2}=-\frac{v}{2}+\frac{1}{2} \sqrt{v^{2}+4 r}>0 \\
& \lambda_{3}=u^{+}(n) \frac{\partial f_{\mathrm{w}}}{\partial S_{\mathrm{w}}}\left(S_{\mathrm{w}}^{+}(n), 0\right)-v>0
\end{aligned}
$$

The latter being positive follows directly from a consistency condition: all characteristic speeds in the two-phase Buckley-Leverett regime must exceed the speed of the SCF (see also [7]). Only the negative eigenvalue $\lambda_{1}$ is relevant, and we have to verify that the corresponding eigenvector $\vec{e}_{1}$ points into domain $\mathcal{R}$. Indeed, a straightforward computation gives

$\frac{\vec{e}_{1} \cdot \vec{k}}{\vec{e}_{1} \cdot \vec{i}}=\frac{1}{2} \frac{v+\sqrt{v^{2}+4 r}}{(1-\alpha) r}>0$ for all $n \in\{0,1, \ldots, N\}$.

Here $\vec{i}$ and $\vec{k}$ are unit vectors as indicated in figure 4 . This inequality shows that $\vec{e}_{1}$ points in the direction of increasing $u$ and $S_{\mathrm{g}}$. Part of the orbit representing the solution $\left(\mathrm{TW}_{+}\right)$is sketched in figure 4 .

Remark 4.1 An eigenvector corresponding to $\lambda_{3}$ is $\vec{e}_{3}=(0,1,0)$. Indeed, a solution of $\left(\mathrm{TW}_{+}\right)$is $(u=$ $\left.u^{+}(n), S_{\mathrm{w}}, S_{\mathrm{g}}=0\right)$ with $S_{\mathrm{w}}$ satisfying

$S_{\mathrm{w}}^{\prime}=u^{+}\left(f_{\mathrm{w}}\left(S_{\mathrm{w}}\right)-f_{\mathrm{w}}\left(S_{\mathrm{w}}^{+}\right)\right)-v\left(S_{\mathrm{w}}-S_{\mathrm{w}^{+}}\right)$.

Let us now turn to the full solution in $\mathcal{R}$. For a fixed $n$, with corresponding curve $\Gamma(n)$, we solve equations $\left(\mathrm{TW}_{+_{-}}\right)$with points from $\Gamma(n)$ as initial condition. Because $u^{\prime}<0$, the solution orbit will move into $\mathcal{R}$. As a first observation, we note that any such orbit cannot leave $\mathcal{R}$ through the side

$\mathbf{S}=\left\{\left(u, S_{\mathrm{w}}, S_{\mathrm{g}}\right): u^{+}<u<1, S_{\mathrm{w}}+S_{\mathrm{g}}=1\right\}$.

Proposition 4.1 Any solution $\left(u(\eta), S_{\mathrm{w}}(\eta), S_{\mathrm{g}}(\eta)\right)$ of $\left(T W_{+}\right)$that belongs to the interior of $\mathcal{R}$ for some $\eta=$ $\eta_{o}>0$, cannot exit $\mathcal{R}$ through $\mathbf{S}$ for $\eta>\eta_{o}$.

Proof We argue by contradiction. Suppose there exists $\eta_{1}>\eta_{o}$ such that $\left(u(\eta), S_{\mathrm{w}}(\eta), S_{\mathrm{g}}(\eta)\right) \in \operatorname{int}(\mathcal{R})$ for $\eta<\eta_{1}$ and $\left(u\left(\eta_{1}\right), S_{\mathrm{w}}\left(\eta_{1}\right), S_{\mathrm{g}}\left(\eta_{1}\right)\right) \in \mathbf{S}$. Then at $\eta=\eta_{1}$ we must have

$$
\frac{\mathrm{d}}{\mathrm{d} \eta}\left(\begin{array}{c}
u \\
S_{\mathrm{w}} \\
S_{\mathrm{g}}
\end{array}\right) \cdot\left(\begin{array}{l}
0 \\
1 \\
1
\end{array}\right)=\left(S_{\mathrm{w}}+S_{\mathrm{g}}\right)^{\prime} \geq 0
$$

However, considering $\left(\mathrm{TW}_{+}\right)$at $\eta_{1}$ and the fact that $S_{\mathrm{w}}+S_{\mathrm{g}}=f_{\mathrm{w}}+f_{\mathrm{g}}=1$ we find

$$
\begin{aligned}
\left(S_{\mathrm{w}}+S_{\mathrm{g}}\right)^{\prime} & =-v-u^{+} f_{\mathrm{w}}^{+}+v S_{\mathrm{w}}^{+}+u^{+} \\
& =u^{+} f_{\mathrm{o}}^{+}-v S_{\mathrm{o}}^{+} \\
& =f_{\mathrm{o}}^{-}-v S_{\mathrm{o}}^{-},
\end{aligned}
$$

where we used $S_{\mathrm{g}}^{+}=0$ and the oil mass balance from (RH) .

We claim that

$f_{\mathrm{o}}^{-}-v S_{\mathrm{o}}^{-}<0 \quad$ for all $\left(S_{\mathrm{w}}^{-}, S_{\mathrm{g}}^{-}\right) \in \ell$,

which would contradict Eq. (40) and complete the proof. Any point $B \in \ell$ is the end point of a fast rarefaction originating from point $A$. In terms of the oil saturation, this rarefaction satisfies, with $\xi=x / t$,

$-\xi \frac{\mathrm{d} S_{\mathrm{o}}}{\mathrm{d} \xi}+\frac{\mathrm{d} f_{\mathrm{o}}}{\mathrm{d} \xi}=0$ for $0<\xi<v$.

Integrating this expression gives

$-v S_{\mathrm{o}}^{-}+f_{\mathrm{o}}^{-}+\int_{0}^{v} S_{\mathrm{o}}(\xi) \mathrm{d} \xi=0$,

which directly implies inequality (41).

Remark 4.2 Equation (42) expresses the oil balance in the steam region. In terms of $x$ and $t$, we have

$\int_{0}^{v t} S_{\mathrm{o}}(x, t) \mathrm{d} x+\left(f_{\mathrm{o}}^{-}-v S_{\mathrm{o}}^{-}\right) t=0$ for all $t>0$,

where $\left(f_{\mathrm{o}}^{-}-v S_{\mathrm{o}}^{-}\right)$denotes the oil flux with respect to the moving front.

Thus, selecting a point on the curve $\Gamma(n)$, the corresponding solution orbit leaves $\mathcal{R}$ through one of the following exit sets (see also figure 4):

$$
\begin{aligned}
& \mathrm{R}(\mathrm{red}) \quad:=\left\{S_{\mathrm{w}}=0\right\} \cup\left\{u=u^{+}, 0<S_{\mathrm{w}}<S_{\mathrm{w}}^{+}\right\} \\
& \mathrm{B} \text { (blue) }:=\left\{S_{\mathrm{g}}=0,0<S_{\mathrm{w}}<S_{\mathrm{w}}^{+}\right\} \\
& \mathrm{Y}\left(\text { yellow) }:=\left\{u=u^{+}, S_{\mathrm{w}}^{+}<S_{\mathrm{w}}<1\right\}\right. \\
& \mathrm{G}(\text { green }):=\left\{S_{\mathrm{g}}=0, S_{\mathrm{w}}^{+}<S_{\mathrm{w}}<1\right\}
\end{aligned}
$$

A point on $\Gamma(n)$ is now colored red, blue, yellow or green depending on the exit set of the orbit. For large $N$ and for a large number of points on $\Gamma(n)$, we cover in this way the front triangle below $\ell$ with these four colors. The point where they meet, denoted by $C=\left\{u=1, S_{\mathrm{w}}(C), S_{\mathrm{g}}(C)\right\}$, and the path through it determine the unique orbit (traveling wave) through $\mathcal{R}$. For the simplified expressions (37) and (38) and with

$v=0.4, r=20$ and $\alpha=0.4$, 


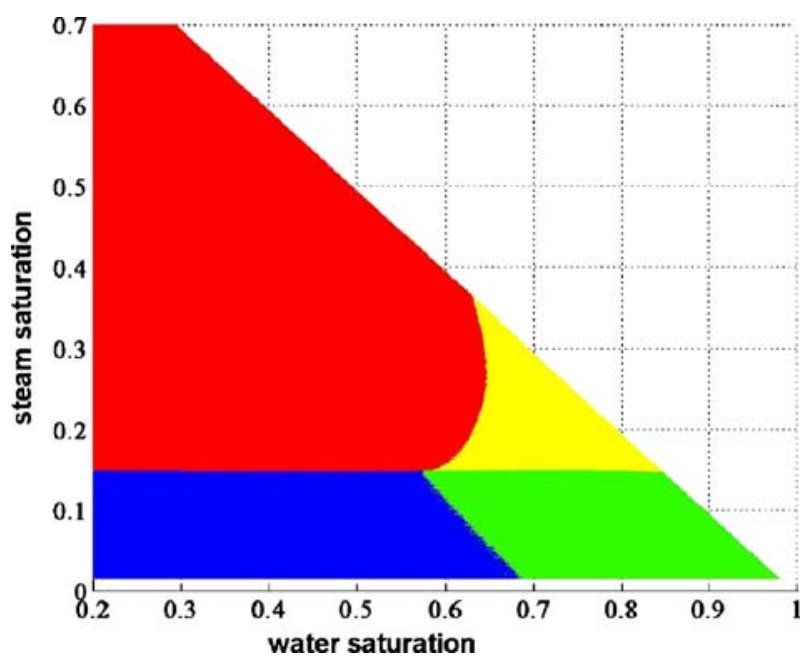

Figure 5 Color distribution in saturation triangle at $\mathbf{T}$.

we computed the color distribution. The result is shown in figure 5, where $S_{\mathrm{w}}(C)=0.582149$ and $S_{\mathrm{g}}(C)=$ 0.149027 . From the orbits for $\eta<0$ and $\eta>0$, we obtain:

$S_{\mathrm{w}}^{-}=0.107351, S_{\mathrm{g}}^{-}=0.789397, S_{\mathrm{o}}^{-}=0.103252$

$S_{\mathrm{w}}^{+}=0.698487, S_{\mathrm{o}}^{+}=0.301513, u^{+}=0.610082$

and

$$
\Lambda(r)=r \int_{0}^{\infty} S_{\mathrm{g}}(\eta) \mathrm{d} \eta=0.649858 .
$$

\section{Towards thermodynamic equilibrium}

The parameter $r$ in Eqs. (20)-(22) describes the finite rate condensation process. On physical grounds, one expects that the limit $r \rightarrow \infty$ will bring the process in thermodynamic equilibrium with either $T=1$, meaning boiling point temperature, or $S_{\mathrm{g}}=0$, with all steam condensing at the SCF. Below we demonstrate this behavior for traveling waves satisfying $(\mathrm{TW}),(\mathrm{RH})$ and (E). Introducing the notation $S_{i}=S_{i}(\eta ; r)$ and $u(\eta ; r)$, we show that

$\lim _{r \rightarrow \infty} r(1-T(\eta)) S_{\mathrm{g}}(\eta ; r)=\Lambda(\infty) \delta(\eta)$,

where $\Lambda(\infty)$ denotes the limiting-instantaneous condensation rate and $\delta$ the Dirac measure at $\eta=0$. Integrating Eq. (43) and using Eq. (25) give

$\Lambda(\infty)=\lim _{r \rightarrow \infty} r \int_{0}^{\infty} S_{\mathrm{g}}(\eta ; r) \mathrm{d} \eta$, which is in agreement with condensation rate (36) in the hyperbolic limit. Replacing the condensation terms in Eqs. (33) by (43) results in the base case discussed in [7].

In terms of the orbit in figure 2, the large $r$ limit means that the point $C$ shifts towards the bottom of $\mathcal{R}$ and that the solution for $\eta>0$ lies entirely in the bottom set $\left\{S_{\mathrm{g}}=0\right\}$.

To demonstrate Eq. (43), we integrate the $u$ equation from (TW) in $\mathbb{R}$. This gives for any $r>0$

$$
\begin{aligned}
1-u^{+}(r) & =(1-\alpha) r \int_{\mathbb{R}}(1-T(\eta)) S_{\mathrm{g}}(\eta ; r) \mathrm{d} \eta \\
& =(1-\alpha) r \int_{\mathbb{R}^{+}} S_{\mathrm{g}}(\eta ; r) \mathrm{d} \eta>0 .
\end{aligned}
$$

Using conditions $\mathrm{RH}$, we find

$$
\begin{aligned}
1>u^{+}(r) & =1-(1-\alpha)\left(f_{\mathrm{g}}^{-}-v S_{\mathrm{g}}^{-}\right) \\
& >\alpha+(1-\alpha) v S_{\mathrm{g}}^{-}>\alpha .
\end{aligned}
$$

Hence

$0<\int_{\mathbb{R}^{+}} S_{\mathrm{g}}(\eta ; r) \mathrm{d} \eta<\frac{1}{r}$ for all $r>0$.

The $S_{\mathrm{g}}$-equation in (TW) implies the existence of a constant $\mathcal{L}>0$ such that

$\left|S_{\mathrm{g}}^{\prime}(\eta ; r)\right| \leq \mathcal{L}$ for all $\eta, r>0$.

Hence from Eqs. (45) and (46),

$\lim _{r \rightarrow \infty} S_{\mathrm{g}}(\eta ; r)=0 \quad$ uniformly in $\quad \eta \geq 0$.

We want to use this convergence in the $S_{\mathrm{g}}$-equation to control $S_{\mathrm{g}}^{\prime}(\eta ; r)$ as $r \rightarrow \infty$. For this purpose, we first write

$S_{\mathrm{g}}^{\prime}=u f_{\mathrm{g}}-v S_{\mathrm{g}}-\frac{1}{1-\alpha}\left(u-u^{+}\right)$.

Multiplying this equation by any test function $\varphi \in$ $C_{o}^{\infty}\left(\mathbb{R}^{+}\right)$and integrating the result in $\mathbb{R}^{+}$give

$$
\begin{aligned}
-\int_{\mathbb{R}^{+}} S_{g} \varphi^{\prime} \mathrm{d} \eta= & \int_{\mathbb{R}^{+}}\left(u f_{\mathrm{g}}-v S_{\mathrm{g}}\right) \varphi \mathrm{d} \eta \\
& -\frac{1}{1-\alpha} \int_{\mathbb{R}^{+}}\left(u-u^{+}\right) \varphi \mathrm{d} \eta .
\end{aligned}
$$

In this expression, we send $r \rightarrow \infty$ and use Eq. (47). This yields

$\lim _{r \rightarrow \infty} \int_{\mathbb{R}^{+}}\left(u(\eta ; r)-u^{+}(r)\right) \varphi(\eta) \mathrm{d} \eta=0$

for all $\varphi \in C_{\mathrm{o}}^{\infty}\left(\mathbb{R}^{+}\right)$. 
Once more, we consider the $u$-equation, which we multiply by $\psi \in C_{\mathrm{o}}^{\infty}\left(\mathbb{R}^{+}\right)$and integrate in $\mathbb{R}^{+}$to find

$$
\begin{array}{r}
\int_{\mathbb{R}^{+}}\left(u(\eta ; r)-u^{+}(r)\right) \psi^{\prime}(\eta) \mathrm{d} \eta= \\
(1-\alpha) r \int_{\mathbb{R}^{+}} S_{\mathrm{g}}(\eta ; r) \psi(\eta) \mathrm{d} \eta .
\end{array}
$$

Because $\psi^{\prime} \in C_{\mathrm{o}}^{\infty}\left(\mathbb{R}^{+}\right)$as well, we can use Eq. (49) and obtain

$\lim _{r \rightarrow \infty} r \int_{\mathbb{R}^{+}} S_{g}(\eta ; r) \psi(\eta) \mathrm{d} \eta=0$ for all $\quad \psi \in C_{\mathrm{o}}^{\infty}\left(\mathbb{R}^{+}\right)$,

which implies

$\lim _{r \rightarrow \infty} r S_{\mathrm{g}}(\eta ; r)=0, \quad$ pointwisely in $\quad \eta>0$.

To see this, we use the following argument. For each $n \in \mathbb{N}, n>1$, the interval $\left(0, \frac{1}{n}\right)$ must contain a point $\eta_{n}$ where $r S_{\mathrm{g}}\left(\eta_{n} ; r\right) \rightarrow 0$ as $r \rightarrow \infty$. This is a direct consequence of Eq. (50). Hence, for $\eta=\eta_{n}$ and $\varepsilon>0$, there exists $r^{*}>0$ such that

$r S_{\mathrm{g}}\left(\eta_{n} ; r\right)<\varepsilon$ for all $r>r^{*}$.

Using this and Eq. (48), we have that $S_{\mathrm{g}}\left(\eta_{n} ; r\right)$ becomes small with $S_{\mathrm{g}}^{\prime}\left(\eta_{n} ; r\right)<0$, due to the quadratic terms in $f_{\mathrm{g}}$ for $r$ sufficiently large. Hence

$r S_{\mathrm{g}}(\eta ; r)<\varepsilon$ for all $r>r^{*}$ and $\eta \geq \eta_{n}$,

implying statement (51).

Thus, we have shown that

$\lim _{r \rightarrow \infty} r(1-T(\eta)) S_{\mathrm{g}}(\eta ; r)=0, \quad$ pointwisely in $\quad \mathbb{R} \backslash\{0\}$ and

$\Lambda(r)=r \int_{\mathbb{R}}(1-T(\eta)) S_{\mathrm{g}}(\eta ; r) \mathrm{d} \eta=\frac{1-u^{+}(r)}{1-\alpha}<1$

for all $r>0$. This establishes Eq. (43) provided

$\Lambda(r)=\frac{1-u^{+}(r)}{1-\alpha}=\left(f_{\mathrm{g}}^{-}-v S_{\mathrm{g}}^{-}\right)(r) \quad($ from $\mathrm{RH})$

remains strictly positive for all $r>0$. We verified the behavior of $\Lambda(r)$ numerically. Computational results show that $\Lambda(r)$ depends only slightly on $r$ and changes from $\Lambda(r)=0.6476$ for $r=1.95313 \times 10^{-3}$ to $\Lambda(r)=$ 0.6499 for $r=4096$. In the same range, $S_{\mathrm{g}}^{-}$changes from 0.80993 to 0.789344 (see (52)). Note that the four-color point $C$ from figure 2 , with $S_{\mathrm{w}}=S_{\mathrm{w}}(C)$

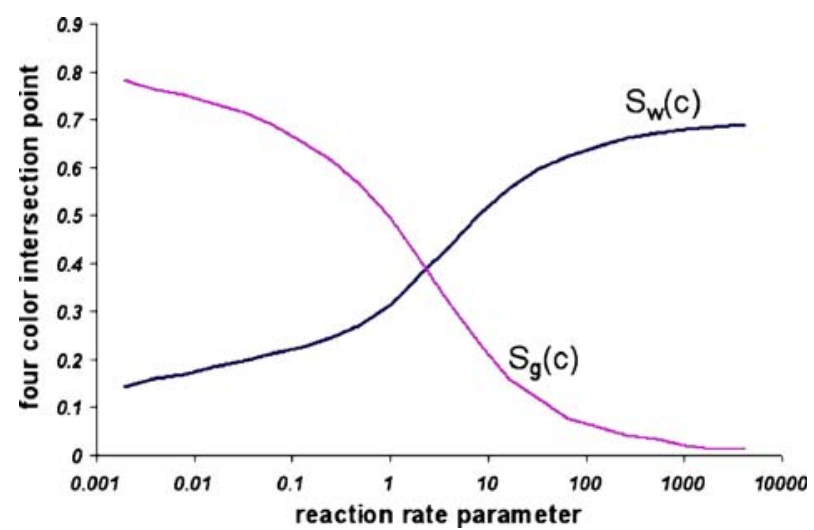

Figure 6 The four-color intersection point $C$ depends strongly on the rate parameter $r$. The effect on the global solution is not zero, but extremely small.

and $S_{\mathrm{g}}=S_{\mathrm{g}}(C)$ changes significantly with $r$. This is shown in figure 6 . However, the quantities describing the global solution such as the downstream velocity $u^{+}$ and the saturation values $S_{\mathrm{w}}^{-}, S_{\mathrm{g}}^{-}$and $S_{\mathrm{w}}^{+}$depend only very weakly on $r$. This is illustrated in figure 7 for the upstream saturations.

\section{A realistic case and comparison to previous results}

In the previous sections, we demonstrate the use of traveling waves to obtain the physically correct saturation and total Darcy velocity values at the SCF. The emphasis was on the principle of construction. Therefore, we disregarded various (non-trivial) technicalities, such as temperature-dependent viscosities (we still disregard the $T$ dependence of the gas viscosity) and saturationdependent capillary diffusion coefficients, and we disregarded the realistic values of the physical parameters as given in table 1 .

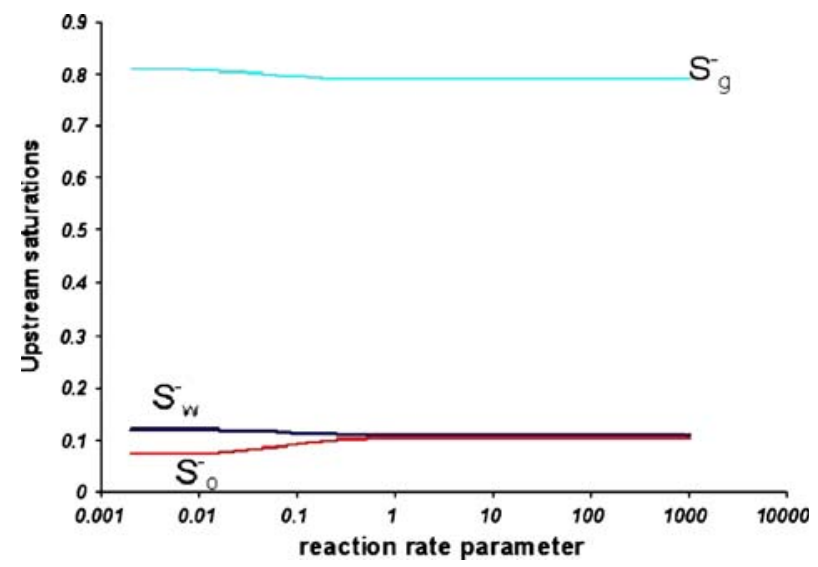

Figure 7 Upstream saturations depend only weakly on the reaction rate parameter $r$. 
In this section, we are going to carry out the construction in a more realistic setting with the aim to explicit the influence of the finite condensation rate under more practical circumstances. We also give a comparison to the results in [7], where we considered several extensions, but all under equilibrium conditions.

With reference to table 1 , we introduce

- Temperature-dependent viscosities, yielding the temperature-dependent viscosity ratios

$M_{\mathrm{ow}}=\frac{\mu_{\mathrm{o}}}{\mu_{\mathrm{w}}}$ and $M_{\mathrm{gw}}=\frac{\mu_{\mathrm{g}}}{\mu_{\mathrm{w}}}$.

- Brooks-Corey capillary pressures (see [1]), yielding additional terms in the expressions for water and gas discharge [see Eqs. (14)-(17)].

- Different relative permeabilities. As in [7], we consider fourth-order power-law expressions as well as unnormalized Stone I formulas (see [9, 17]):

$$
\begin{aligned}
& k_{\mathrm{rw}}=k_{\mathrm{rw}}\left(S_{\mathrm{we}}\right)=\frac{1}{2} S_{\mathrm{we}}^{\frac{2+3 \lambda_{\mathrm{s}}}{\lambda \mathrm{s}}} \\
& k_{\mathrm{rg}}=k_{\mathrm{rg}}\left(S_{\mathrm{ge}}\right)=\left(1-S_{\mathrm{ge}}\right)^{2}\left(1-S_{\mathrm{ge}}^{\frac{2+\lambda \mathrm{s}}{\lambda \mathrm{s}}}\right) \\
& k_{\mathrm{ro}}=\frac{k S_{\mathrm{o}}\left(1-S_{\mathrm{wc}}\right) k_{\mathrm{rg}}\left(S_{\mathrm{we}}\right) k_{\mathrm{rw}}\left(S_{\mathrm{ge}}\right)}{\left(1-S_{\mathrm{w}}\right)\left(1-S_{\mathrm{wc}}-S_{\mathrm{g}}\right)}
\end{aligned}
$$

where $S_{\mathrm{we}}=\frac{S_{\mathrm{w}}-S_{\mathrm{wc}}}{1-S_{\mathrm{wc}}}, S_{\mathrm{ge}}=\frac{1-S_{\mathrm{g}}-S_{\mathrm{wc}}}{1-S_{\mathrm{wc}}}$. Using a gas saturation-dependent residual oil saturation is for the steam drive problem an unnecessary complication, and therefore, we assume that the residual oil saturation is always zero.

With these extensions, the model equations in full dimensional form are

$\frac{\partial u}{\partial x}=-(1-\alpha) \frac{q}{\rho_{\mathrm{g}}}$,

$\varphi \frac{\partial S_{\mathrm{w}}}{\partial t}+\frac{\partial}{\partial x}\left(u f_{\mathrm{w}}-\mathcal{D}_{\mathrm{ww}} \frac{\partial S_{\mathrm{w}}}{\partial x}-\mathcal{D}_{\mathrm{wg}} \frac{\partial S_{\mathrm{g}}}{\partial x}\right)=\frac{q}{\rho_{\mathrm{w}}}$,

$\varphi \frac{\partial S_{\mathrm{g}}}{\partial t}+\frac{\partial}{\partial x}\left(u f_{\mathrm{g}}-\mathcal{D}_{\mathrm{gw}} \frac{\partial S_{\mathrm{w}}}{\partial x}-\mathcal{D}_{\mathrm{gg}} \frac{\partial S_{\mathrm{g}}}{\partial x}\right)=-\frac{q}{\rho_{\mathrm{g}}}$.

Note that the fractional flow functions $f_{\alpha}$, and hence the diffusivities $D_{\alpha \beta}$, are discontinuous across the SCF. This is due to the temperature dependence of the mobility ratios.

Using Eq. (56) to estimate $q$ from Eqs. (57) and (58), substituting Eq. (1) in Eq. (56) and applying the scalings (18) and (19) gives

$$
\frac{\partial u}{\partial x}=-\frac{q_{\mathrm{b}} L}{u_{\mathrm{inj}}}(1-\alpha)(1-T) S_{\mathrm{g}},
$$

$$
\begin{aligned}
& \frac{\partial S_{\mathrm{w}}}{\partial t}+\frac{\partial}{\partial x}\left(u f_{\mathrm{w}}-\mathcal{D}^{*}\left(\mathcal{J}_{\mathrm{ww}} \frac{\partial S_{\mathrm{w}}}{\partial x}+\mathcal{J}_{\mathrm{wg}} \frac{\partial S_{\mathrm{g}}}{\partial x}\right)\right)=\frac{\alpha}{\alpha-1} \frac{\partial u}{\partial x}, \\
& \frac{\partial S_{\mathrm{w}}}{\partial t}+\frac{\partial}{\partial x}\left(u f_{\mathrm{g}}-\mathcal{D}^{*} \frac{\partial}{\partial x}\left(\mathcal{J}_{\mathrm{gw}} \frac{\partial S_{\mathrm{w}}}{\partial x}+\mathcal{J}_{\mathrm{gg}} \frac{\partial S_{\mathrm{g}}}{\partial x}\right)\right)=-\frac{1}{\alpha-1} \frac{\partial u}{\partial x},
\end{aligned}
$$

Here

$\mathcal{D}^{*}=\frac{\sigma}{\mu_{\mathrm{w}}^{*} L u_{\mathrm{inj}}} \sqrt{k \varphi}\left(\frac{\frac{1}{2}-S_{\mathrm{wc}}}{1-S_{\mathrm{wc}}}\right)^{1 / \lambda_{\mathrm{s}}}$

where again $\mu_{\mathrm{w}}^{*}$ is an appropriately chosen characteristic water viscosity (here the viscosity at the initial reservoir temperature) and

$$
\begin{aligned}
& \mathcal{J}_{\mathrm{ww}}=\frac{\mu_{\mathrm{w}}^{*}}{\mu_{\mathrm{w}}} \frac{\lambda_{\mathrm{s}} f_{\mathrm{w}}}{1-S_{\mathrm{wc}}}\left(\frac{k_{\mathrm{ro}}}{M_{\mathrm{ow}}}+\frac{k_{\mathrm{rg}}}{M_{\mathrm{gw}}}\right)\left(\frac{S_{\mathrm{w}}-S_{\mathrm{wc}}}{1-S_{\mathrm{wc}}}\right)^{-1 / \lambda_{\mathrm{s}}-1}, \\
& \mathcal{J}_{\mathrm{wg}}=-\frac{\mu_{\mathrm{w}}^{*}}{\mu_{\mathrm{w}}} \frac{\lambda_{\mathrm{s}} f_{\mathrm{w}}}{1-S_{\mathrm{wc}}} \frac{k_{\mathrm{rg}}}{M_{\mathrm{gw}}}\left(\frac{1-S_{\mathrm{g}}-S_{\mathrm{wc}}}{1-S_{\mathrm{wc}}}\right)^{-1 / \lambda_{\mathrm{s}}-1}, \\
& \mathcal{J}_{\mathrm{gw}}=-\frac{\mu_{\mathrm{w}}^{*}}{\mu_{\mathrm{w}}} \frac{\lambda_{\mathrm{s}} f_{\mathrm{g}}}{1-S_{\mathrm{wc}}} k_{\mathrm{rw}}\left(\frac{S_{\mathrm{w}}-S_{\mathrm{wc}}}{1-S_{\mathrm{wc}}}\right)^{-1 / \lambda_{\mathrm{s}}-1}, \\
& \mathcal{J}_{\mathrm{gg}}=\frac{\mu_{\mathrm{w}}^{*}}{\mu_{\mathrm{w}}} \frac{\lambda_{\mathrm{s}} f_{\mathrm{g}}}{1-S_{\mathrm{wc}}}\left(\frac{k_{\mathrm{ro}}}{M_{\mathrm{ow}}}+k_{\mathrm{rw}}\right)\left(\frac{1-S_{\mathrm{g}}-S_{\mathrm{wc}}}{1-S_{\mathrm{wc}}}\right)^{-1 / \lambda_{\mathrm{s}}-1} .
\end{aligned}
$$

Introducing the reciprocal Peclet number $\varepsilon$ and the dimensionless rate constant $r$ as

$$
\begin{aligned}
& \varepsilon:=\frac{\sigma}{\mu_{\mathrm{w}}^{*} L u_{\mathrm{inj}}} \sqrt{\mathcal{K} \varphi}\left(\frac{\frac{1}{2}-S_{\mathrm{wc}}}{1-S_{\mathrm{wc}}}\right)^{1 / \lambda_{\mathrm{s}}}, \\
& r:=q_{\mathrm{b}} \frac{\sigma}{\mu_{\mathrm{w}}^{*} u_{\mathrm{inj}}^{2}} \sqrt{\mathcal{K} \varphi}\left(\frac{\frac{1}{2}-S_{\mathrm{wc}}}{1-S_{\mathrm{wc}}}\right)^{1 / \lambda_{\mathrm{s}}}=\varepsilon q_{\mathrm{b}} \frac{L}{u_{\mathrm{inj}}},
\end{aligned}
$$

applying a traveling wave coordinate transformation $\eta=(x-v t) / \varepsilon$ and integrating the resulting equations leads to the system, with $-\infty<\eta<\infty$,

$u^{\prime}=-r(1-\alpha)(1-T) S_{\mathrm{g}}$,

$\mathcal{J}_{\mathrm{ww}} S_{\mathrm{w}}^{\prime}+\mathcal{J}_{\mathrm{wg}} S_{\mathrm{g}}^{\prime}=u f_{\mathrm{w}}-v S_{\mathrm{w}}-\frac{\alpha(1-u)}{1-\alpha}-\left(u f_{\mathrm{w}}-v S_{\mathrm{w}}\right)^{-}$

$\mathcal{J}_{\mathrm{gw}} S_{\mathrm{w}}^{\prime}+\mathcal{J}_{\mathrm{gg}} S_{\mathrm{g}}^{\prime}=u f_{\mathrm{g}}-v S_{\mathrm{g}}-\frac{u-1}{1-\alpha}-\left(u f_{\mathrm{g}}-v S_{\mathrm{g}}\right)^{-},$.

Note that $q_{\mathrm{b}} L / u_{\text {inj }}$, the Damkohler number, is considered to be of the same order of magnitude as the Peclet number $1 / \varepsilon$. As before, we look for solutions 
satisfying boundary conditions $(\mathrm{BC})$ subject to the constraints (E) and (RH). Note that Eqs. (65)-(67) reduce to (TW) when $\mathcal{J}_{\text {wg }}=\mathcal{J}_{\text {gw }}=0$ and $\mathcal{J}_{\text {ww }}=\mathcal{J}_{\text {gg }}=1$.

Equations (65) and (67) can be rearranged to explicit expressions for $S_{w}^{\prime}$ and $S_{g}^{\prime}$ if the determinant

$$
\left|\begin{array}{cc}
\mathcal{J}_{w w} & \mathcal{J}_{w g} \\
\mathcal{J}_{g w} & \mathcal{J}_{g g}
\end{array}\right| \neq 0
$$

It is straightforward to verify these conditions. Details are omitted. The rearranged equations, with explicit $S_{w}^{\prime}$ and $S_{g}^{\prime}$ are used in the numerical procedure.

\subsection{Procedure for determining the traveling wave orbit}

The procedure to find the traveling wave describing the processes in the SCF for the realistic case is slightly different from the procedure used in the previous sections. The reason is that we need a more robust method for solutions with small values of the reaction rate parameter $r$, for which the four color point (see figure 5) approaches the line $\ell$ satisfying condition (30). Our aim is to find the orbit $D--C--B(n)$ satisfying Eqs. (65)-(67), satisfying the Rankine-Hugoniot conditions and condition (30). First we choose as an initial guess a value $n^{\prime}$ and determine as in (39) the corresponding value of $S_{\mathrm{w}}\left(n^{\prime}\right)$. Subsequently, we apply condition (30) to determine $S_{\mathrm{g}}\left(n^{\prime}\right)$ and thus point $B\left(n^{\prime}\right)$ on $\ell$ (see figure 8 ). Subsequently, we determine from the left (upstream) values of $\left(u^{-}=1, S_{\mathrm{w}}^{-}, S_{\mathrm{g}}^{-}\right)$ at $B\left(n^{\prime}\right)$ the right (downstream) values $\left(u^{+}, S_{\mathrm{w}}^{+}, S_{\mathrm{g}}^{+}\right)$, i.e. point $D^{\prime}$ in figure 8 , using the Rankine-Hugoniot conditions (29). At the equilibrium point $B\left(n^{\prime}\right)$, we

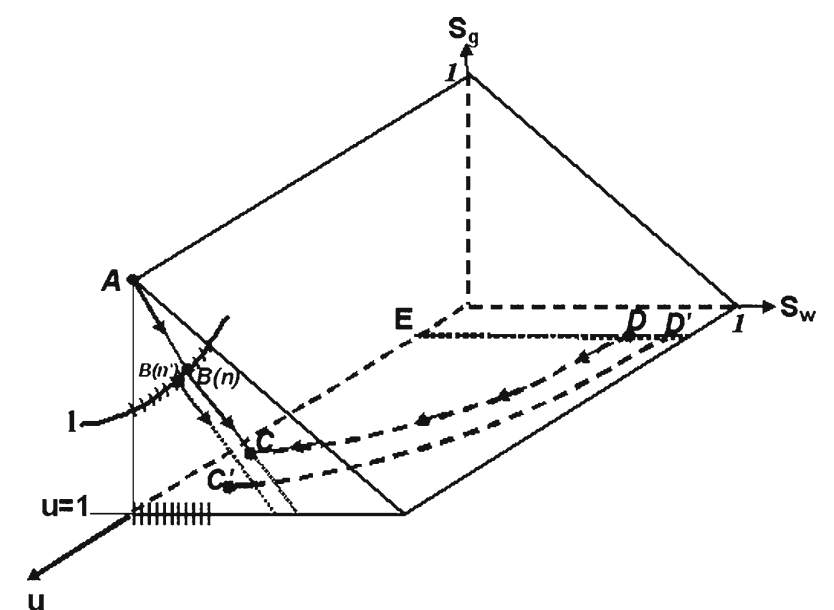

Figure 8 The orbits from $\left(u^{-}=1, S_{\mathrm{g}}^{-}, S_{\mathrm{w}}^{-}\right)$in the plane $u=1$ and orbits in the negative $\eta$ direction from $\left(u^{+}, S_{\mathrm{g}}^{+}, S_{\mathrm{w}}^{+}\right)$that intersect in the $u=1$ plane at $C$ are found using bissection (see text). determine the eigenvector to obtain the first point on the orbit away from it. We use this point as initial condition for the rearranged conditions (65)-(67) in the hot-upstream-region, and we determine as before the corresponding orbit until we hit $S_{\mathrm{g}}=0$. We compute the orbit emanating from $B\left(n^{\prime}\right)$ using Eq. (65)-(67) in the positive $\eta$ direction until we hit the $S_{\mathrm{g}}=0$ axis. We call this orbit $\Gamma\left(n^{\prime}\right)$. At the downstream point $D^{\prime}=$ $\left(u^{+}, S_{\mathrm{w}}^{+}, S_{\mathrm{g}}^{+}\right)$, we apply a similar procedure; that is, we determine the eigenvector pointing into the domain $\mathcal{R}$ to obtain the first point away from this equilibrium point. Then we solve the rearranged Eqs. (65)-(67) in the cold-downstream region, this time in the negative $\eta$ direction until we hit the $u=1$ plane with values $\left(S_{\mathrm{w}}\left(C^{\prime}\right), S_{\mathrm{g}}\left(C^{\prime}\right)\right)$ (see figure 8 ). We choose a second guess, e.g. $\widetilde{n}^{\prime}$, to start at a new point $B\left(\widetilde{n}^{\prime}\right)$ to the right or left with respect to $B\left(n^{\prime}\right)$ depending on whether $S_{\mathrm{w}}\left(C^{\prime}\right)$ was to the right or to the left of $\Gamma\left(n^{\prime}\right)$ and repeat the procedure above. For the sequence of points $n^{\prime}, \widetilde{n}^{\prime}, \ldots$, we use a bisection routine until we approximate the complete orbit $B(n)-C-D$, with $\left(S_{\mathrm{w}}(C), S_{\mathrm{g}}(C)\right)$ on the orbit $\Gamma(n)$.

\subsection{Results}

Figure 9 shows the upstream oil saturation as a function of the reaction rate parameter. We distinguish four cases where we use either Stone I expressions (53-55) (ST) or power-law expressions (PL) for the relative permeability and either a constant capillary diffusion (D) (see table 1) or a saturation-dependent capillary diff usion coefficient (Pc) (see (17)). Figure 9 also shows, for each of these cases, the oil saturation obtained when

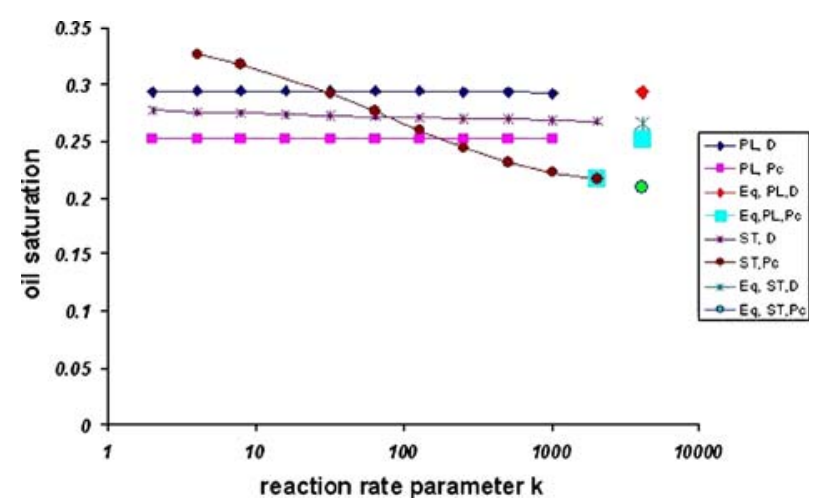

Figure 9 Upstream oil saturation as a function of the reaction rate parameter. There are four cases. $P L=$ power-law relative permeabilities, $S T=$ Stone I relative permeabilities, $D=$ constant capillary diffusion, $P c=$ saturation-dependent capillary diffusion. The points $(E q, P L, D),(E q, P L, P c),(E q, S T, D)$ and $(E q, P L, P c)$ correspond to the solutions obtained for thermodynamic equilibrium. 
thermodynamic equilibrium is assumed [7]. As to be expected from Section 5, the oil saturation values are about equal to the values obtained for large reaction rate parameters. It is evident that in all cases, the global solution depends strongly on the capillary pressure behavior for the same relative permeability expressions. The numerical results, shown in figure 9, suggest that the global solution in terms of the values $S_{\mathrm{w}}^{-}, S_{\mathrm{g}}^{-}, S_{\mathrm{w}}^{+}, u^{+}$ is very insensitive to the reaction rate parameter except for the case where we combine saturation-dependent capillary pressures and Stone I expressions for the relative permeabilities.

\section{Conclusions}

1. A hyperbolic model for steam displacement of oil was extended with a finite rate condensation model in the transition zone.

2. The traveling wave describing the shock solution is a saddle-to-saddle connection. Consequently, the global solution depends on the details of the condensation model within the shock.

3. Using color coding, it has been shown numerically that, given the parameters describing the condensation process, there is a unique set of values $S_{\mathrm{w}}^{-}, S_{\mathrm{g}}^{-}, S_{\mathrm{w}}^{+}, u^{+}$, etc., for which a traveling wave exists (see figure 5).

4. A proof was given that the solution with an infinite reaction rate parameter tends to the solution obtained when thermodynamic equilibrium is assumed.

5. The numerical solutions show that there is a dependence of the global solution on the reaction rate parameter. For power-law relative permeabilities, this dependence is very weak.

6. The procedure described can also be used for a realistic set of input variables. When we combine Stone I relative permeabilities with saturationdependent capillary pressures, the effect of the reaction rate parameter is significant.

Acknowledgement We acknowledge D. Marchesin [Instituto de Matemática Pura é Aplicada, Rio de Janeiro (Brazil)] for his suggestion of color coding the orbits in the triangle $\mathbf{T}$. This work was partially carried out at the Institute for Mathematics and its Application (IMA), Minneapolis, MN.

\section{References}

1. Aziz, K., Settari, A.: Petroleum Reservoir Simulation. Applied Science Publishers, London (1979)
2. Bear, J.: Dynamics of Fluids in Porous Media. Dover Publications, Inc., Dover (1972)

3. Betz, C., Farbar, A., Schmidt, R.: Removing Volatile and Semi-Volatile Contaminants from the Unsaturated Zone by Injection of a Steam Air Mixture. Thomas Telford. Contaminated Soil (1998)

4. Bird, R., Stewart, W., Lightfoot, E.: Transport Phenomena. John-Wiley, New York (1960)

5. Brantferger, R., Pope, G., Sepehrnori, K.: Development of a thermodynamically consistent, fullyImplicit equation of state, ompositional steamfllod simulator. SPE 21253. SPE Symposium Reservoir Simulation, Anaheim (1991)

6. Brooks, R., Corey, A.: Properties of porous media affecting fluid. Flow. J. Irrig. Drain. Div. 6, 61 (1966)

7. Bruining, J., van Duijn, C.: Uniqueness conditions in a hyperbolic model for oil recovery by steamdrive. Comput. Geosci. 4, 65-98 (2000)

8. Chien, M., Yardumian, H., Chung, E., Todd, W.: The formulation of a thermal simulation model in a vectorized, general purpose reservoir simulator. SPE 18418. The SPE Symposium on Reservoir Simulation in Houston (1989)

9. Fayers, F.J., Matthews, J.: Evaluation of normalized Stone's methods for estimating three-phase relative permeabilities. Soc. Pet. Eng. J. 224-232 (1984)

10. Fayers, F.J., Sheldon, J.: The effect of capillary pressure and gravity on two-phase fluid flow in a porous medium. Trans. AIME 216, 147 (1959)

11. Goderij, R., Bruining, J., Molenaar, J.: A fast 3D interface simulator for steam drives. SPE J. 4 (4), 400-408.SPEWestern Regional Meeting (June-1997) 279-289 (SPE-38288) (1999)

12. Grabensetter, J., Li, Y.-K., Collins, D., Nghiem, L.: Stability based switching criterion for adaptive-implicit compositional reservoir simulation. SPE Symposium Reservoir Simulation Anaheim (1991) SPE 21225 (1991)

13. Gümrah, F., Palmgren, C., Bruining, J., Godderij, R.: Steamdrive in a layered reservoir: an experimental and theoretical study. In: Proc. SPE/DOE 8th Symposium on Enhanced Oil Recovery, Tulsa SPE/DOE 24171, 159-167 (1992)

14. Guzman, R., Fayers, F.: Mathematical properties of threephase flow equations. SPE J. 2, 291-300 (1997a)

15. Guzman, R., Fayers, F.: Solutions to the three-phase Buckley-Leverett problem. SPE J. 2, 301-311 (1997b)

16. Hanzlik, E., Mims, D.: Forty years of steam injection in California-evolution of heat management. SPE International Improved Oil Recovery Conference in Asia Kuala Lumpur Malaysia, SPE 84848 (2003)

17. Honarpour, M., Koederitz, L., Harvey, A.: Relative Permeability of Petroleum Reservoirs. Boca Raton, Florida, USA: CRC Press (1986)

18. Hovanessian, S., Fayers, F.: Linear waterflood with gravity and capillary effects. Soc. Pet. Eng. J. I, 32 (1961)

19. Hunt, J., Sitar, N., Udell, K.: Non-aqueous phase liquid transport and clean up: part I, analysis of mechanisms. Water Resour. Res. 24 (8), 1247-1258 (1988a)

20. Hunt, J., Sitar, N., Udell, K.: Non-aqueous phase liquid transport and clean up: part II, experimental studies. Water Resour. Res. 24 (8), 1259-1269 (1988b)

21. Isaacson, E., Marchesin, D., Plohr, B.: Transitional waves for conservation laws. SIAM J. Math. Anal. 21, 837-866 (1990)

22. Isaacson, E., Marchesin, D., Plohr, B., Temple, J.: Multiphase flow models with singular Riemann problems. Math. Appl. Comput. 2, 147-166 (1992)

23. Kaslusky, S., Udell, K.: A theoretical model of air and steam co-injection to prevent the downward migration of 
the DNAPL's during steam enhanced extraction. J. Contam. Hydrol. 55, 213-232 (2002)

24. Kimber, K., Ali, S.F., Puttagunta, V.: New scaling criteria and their relative merits for steam recovery experiments. J. Can. Petrol. Technol. 27, 86-94 (1988)

25. Lax, P.: The formation and decay of shock waves. Am. Math. Monthly 79, 227-241 (1988)

26. Mandl, G., Volek, C.: Heat and mass transport in steamdrive processes. Soc. Pet. Eng. J. 57-79 (1969)

27. Marchesin, D., Schaeffer, D., Shearer, M., Paes-Leme: The classification of $2 \times 2$ systems of non-strictly hyperbolic conservation laws, with application to oil recovery. Comm. Pure Appl. Math. 40, 141-178 (1987)

28. Menegus, D., Udell, K.: A study of steam injection into water saturated porous media, heat transfer in porous media and particle flows. ASME Heat Transf. Div. New York City. 46, 151-157 (1985)

29. Mifflin, R., Watts, J.: A fully coupled, fully implicit simulator for thermal and other complex reservoir processes. SPE 21252 Symposium Reservoir Simulation, Anaheim (1991) $21252(1969)$

30. Naccache, P.: A fully implicit thermal reservoir simulator. SPE 37985 Reservoir Simulatiuon Symposium, Dallas (1997)

31. Oballa, V., Coombe, D., Buchanan, W.: Adaptive-implicit method in thermal simulation. SPE Reserv. Eng. 549-556 (1990)

32. Prats, M.: Thermal Recovery. Dallas: SPE Henry L. Doherty Series, Monograph 7 (1982)

33. Rapoport, L.: Scaling laws for use in design and operation of water-oil flow models. Trans. AIME 204, 143 (1955)

34. Rapoport, L., Leas, W.: Properties of linear waterfloods. Trans. AIME 198, 139-148 (1953)

35. Reid, R., Parausnitz, J., Sherwood, T.: The Properties of Gases and Liquids. New York: McGraw-Hill (1977)
36. Rose, W., Bruce, W.: Evaluation of capillary characters in petroleum reservoir rock. Trans. AIME 186, 127-142 (1949)

37. Schmidt, R., Betz, C., Faerber, A.: LNAPL and DNAPL behaviour during steam injection into the unsaturated zone. Int. Assoc. Hydrol. Sci. Publ. 250, 11-117 (1998)

38. Schmidt, R., Gudbjerg, J., Sonnenborg, T.O., Jensen, K.: Removal of NAPL's from the unsaturated zone using steam: prevention of downsward migration by injecting mixtures of steam and air. J. Contam. Hydrol. 55, 233-260 (2002)

39. Shutler, N.: A one dimensional analytical technique for predicting oil recovery by steam flooding. Soc. Pet. Eng. J. 489498 (1972)

40. Stewart, L., Udell, K.: Mechanisms of residual oil displacement by steam injection. SPE Reserv. Eng. 1233-1242 (1988)

41. Tortike, W., Farouq-Ali, S.: Saturated-steam-property functional correlations for fully implicit thermal reservoir simulation. SPE Reserv. Eng. 4(4), 471-474 (1989)

42. Udell, K.: The thermodynamics of evaporation and condensation in porous media. SPE 10779, California Regional Meeting of the Society of Petroleum Engineers, San Francisco (1982)

43. Willman, B., Valeroy, V., Runberg, G., Cornelius, A.: Laboratory studies of oil recovery by steam injection. J. Petrol. Technol. 681-690 (1961)

44. Wingard, J., Orr, F.: An analytical solution for steam/oil/ water displacements. SPE Adv. Technol. Ser. 2, 167-176 (1994)

45. Yortsos, Y.: Distribution of fluid phases within the steam zone in steam injection processes. Soc. Pet. Eng. J. 458-466 (1984)

46. Yortsos, Y., Gavalas, G.: Heat transfer ahead of moving condensation fronts in thermal oil recovery processes. Int. J. Heat Mass Transfer 25(3), 305-316 (1982) 